\title{
Appendix
}

\section{The European Stroke Initiative (EUSI)}

\section{Members}

Markku Kaste, Helsinki (Finland), Chairman

Julien Bogousslavsky, Lausanne (Switzerland), representing the ENS Otto Busse, Minden (Germany), representing the ESC

Eberhard Deisenhammer, Linz (Austria), representing the EFNS

Hubert Kwiecinski, Warzawa (Poland), representing the EFNS

Tom Skyhoj Olsen, Copenhagen (Denmark), representing the ENS

Jean-Marc Orgogozo, Bordeaux (France), representing the ESC

Werner Hacke, Heidelberg (Germany), Secretary

This supplement issue is approved and endorsed by

- The European Federation of Neurological Societies (EFNS)

- The European Neurological Society (ENS)

- The European Stroke Council (ESC) 